\title{
An Innovate Evaluation Index Method of Electronic Technology Teaching Quality based on Outcomes-based Education
}

\author{
Yang Zhao ${ }^{\mathrm{a}^{*}}$ and $\mathrm{Li} \mathrm{Li}^{\mathrm{b}}$ \\ Department of Mechanical and Electrical Engineering, Guangdong University of Science \& \\ Technology, Dongguan, China \\ azhaoyangmatp@163.com, ${ }^{\mathrm{b}} 65799279 @ q q . c o m$ \\ * The Corresponding Author
}

Keywords: Evaluation Index; Electronic technology; Teaching quality; Outcomes-based education

\begin{abstract}
In the association rules, evaluation index can mine the associated data hidden in the data items or data attributes, and is widely used in communication engineering, efficient management and so on. The innovate evaluation index method has an important influence on the employment and postgraduate entrance examination of college students. With the emergence and development of the outcomes-based education, people expect to predict and evaluate scores by using evaluation index technology. This paper uses evaluation index technology to analyze and excavate a large amount of data. The association rules are used to find the relation among them, and the teaching quality evaluation system is constructed through the improvement of evaluation index.
\end{abstract}

\section{Introduction}

How to predict and evaluate the relationship between students' usual electronic technology performance and passing rate has been one of the problems studied in teaching quality research. Through the excavation of teaching or student data, the internal relationship between data is found. The electronic technology teaching quality is an important symbol to measure ability. How to use evaluation index to correctly evaluate students is a common concern of teachers and students. The electronic technology teaching quality is related to many factors, such as the scores in college entrance examination. This paper uses evaluation index technology to analyze and excavate a large number of data, and uses association rules to find out the relationship between the results of college entrance examination and electronic technology performance by outcomes-based education.

\section{The Innovate Evaluation Index Model}

An evaluation analysis method combining qualitative analysis is studied and the overall influence factors on the evaluation of teaching quality, teaching theoretical knowledge, knowledge development and cultivation of professional ability can be explained by the research on the electronic technology teaching quality evaluation. This evaluation system of electronic technology teaching quality includes teachers' professionalism, work attitude, teaching methods etc.

The teaching quality evaluation index system was initially constructed to judge the electronic technology teaching level. The calculation method of correlation score was as follows:

Let $y$ be a dependent variable, $x_{1}, x_{2}, \ldots, x_{\mathrm{m}}$ is all independent variables, $y_{i}, x_{i 1}, x_{i 2}, \ldots, \mathrm{x}_{i m}(i=1,2, \ldots, n)$ is an independently extracted $n$ group sample. The significant level of the independent variable was selected as the model, and the significant level of the excluded model was as follows,

Step 1. Calculation of deviation matrix $S$,

$$
S=S_{m \times m}=\left(\begin{array}{ccccc}
s_{11} & s_{12} & \cdots & s_{1 m} & s_{1 y} \\
s_{21} & s_{22} & \cdots & s_{2 m} & s_{2 y} \\
\cdots & \cdots & \cdots & \cdots & \cdots \\
s_{m 1} & s_{m 2} & \cdots & s_{m m} & s_{m y}
\end{array}\right)
$$

Step 2. The gradually screened independent variables 
Calculate the contribution of each variable

$V_{j}^{(1)}=\frac{s_{j y}^{2}}{s_{j j}}$

Maximum value

$V_{k_{1}}^{(1)}=\max _{1 \leq j \leq m} V_{j}^{(1)}$

Whether the effect of step 3 is statistically significant or not, then

$$
F=\frac{V_{k_{1}}^{(1)}}{S_{E}^{(1)} /(n-1-1)}
$$$$
S_{E}^{(1)}=S_{T}-V_{k_{1}}^{(1)}
$$

If $F \leq F_{\alpha_{1}}(1, n-1-1)$ all the independent variables were independent of $y$, and the regression equation could not be established.

If $F>F_{\alpha_{1}}(1, n-1-1)$ then $x_{k 1}$ is selected into the model, and $S$ is converted to $S_{m \times(m+1)}^{(1)}$.

$$
\begin{aligned}
& S_{m \times(m+1)}^{(1)}=\left(\begin{array}{ccccc}
s_{11}^{(1)} & s_{12}^{(1)} & \ldots & s_{1 m}^{(1)} & s_{1 y}^{(1)} \\
s_{21}^{(1)} & s_{22}^{(1)} & \ldots & s_{2 m}^{(1)} & s_{2 y}^{(1)} \\
\ldots & \ldots & \ldots & \ldots & \ldots \\
s_{k_{1} 1}^{(1)} & s_{k_{1} 2}^{(1)} & \ldots & s_{k_{1} m}^{(1)} & s_{k_{1} y}^{(1)} \\
\ldots & \ldots & \ldots & \ldots & \ldots \\
s_{m 1}^{(1)} & s_{m 2}^{(1)} & \ldots & s_{m m}^{(1)} & s_{m y}^{(1)}
\end{array}\right) \\
& s_{i j}^{(1)}=\left\{\begin{array}{c}
\frac{s_{k_{1} i}}{s_{k_{1} k_{1}}}\left(i=k_{1}, j \neq k_{1}\right) \\
s_{i j}-\frac{s_{i k_{1}} s_{k_{1} j} j}{s_{k_{1} k_{1}}}\left(i \neq k_{1}, j \neq k_{1}\right) \\
\frac{1}{s_{k_{1} k_{1}}}\left(i=j=k_{1}\right) \\
-\frac{s_{i k_{1}}}{s_{k_{1} k_{1}}}\left(i \neq k_{1}, j=k_{1}\right)
\end{array}\right.
\end{aligned}
$$

Calculate the contribution of each variable according to $S_{m \times(m+1)}^{(1)}$.

$$
\begin{aligned}
& V_{i}^{(2)}=\frac{\left(s_{i y}\right)^{2}}{s_{i i}} \\
& V_{k_{1}}^{(2)}=\frac{\left(s_{k_{1} y}^{(1)}\right)^{2}}{s_{k_{1} k_{1}}^{(1)}}
\end{aligned}
$$

Take the maximum contribution of the independent variables outside the model, that is

$$
\begin{aligned}
& V_{k_{2}}^{(2)}=\max _{j \neq k_{1}} V_{j}^{(2)} \\
& F=\frac{V_{k_{2}}^{(2)}}{S_{E}^{(2)} /(n-2-1)}
\end{aligned}
$$


$S_{E}^{(2)}=S_{T}-V_{k_{2}}^{(2)}$

If $F \leq F_{\alpha_{1}}(1, n-2-1)$ then the optimal regression equation is established.

If $F>F_{\alpha_{1}}(1, n-2-1)$ then the election $x_{k_{2}}$ entry model is

$S_{m \times(m+1)}^{(2)}=\left(\begin{array}{ccccc}s_{11}^{(2)} & s_{12}^{(2)} & \cdots & s_{1 m}^{(2)} & s_{1 y}^{(2)} \\ s_{21}^{(2)} & s_{22}^{(2)} & \cdots & s_{2 m}^{(2)} & s_{2 y}^{(2)} \\ \cdots & \cdots & \cdots & \cdots & \cdots \\ s_{k_{2} 1}^{(2)} & s_{k_{2} 2}^{(2)} & \cdots & s_{k_{2} m}^{(2)} & s_{k_{2} y}^{(2)} \\ \cdots & \cdots & \cdots & \cdots & \cdots \\ s_{m 1}^{(2)} & s_{m 2}^{(2)} & \cdots & s_{m m}^{(2)} & s_{m y}^{(2)}\end{array}\right)$

$$
s_{i j}^{(2)}=\left\{\begin{array}{c}
\frac{s_{k_{2} j}^{(1)}}{s_{k_{2} k_{2}}^{(1)}}\left(i=k_{2}, j \neq k_{2}\right) \\
s_{i j}^{(1)}-\frac{s_{i k_{2}}^{(1)} s_{k_{2} j}^{(1)}}{s_{k_{2} k_{2}}^{(1)}}\left(i \neq k_{2}, j \neq k_{2}\right) \\
\frac{1}{s_{k_{2} k_{2}}^{(1)}}\left(i=j=k_{2}\right) \\
-\frac{s_{i k_{2}}^{(1)}}{s_{k_{2} k_{2}}^{(1)}}\left(i \neq k_{2}, j=k_{2}\right)
\end{array}\right.
$$

\section{Construction and Result Analysis of Outcomes-based Education Achievement Model}

The students at a school are selected as the research objects and each sample contains the scores in college entrance examination. Their scores in college entrance examination are achieved, and the simulation results are selected as the research data source to excavate and analyze the association rules between the results and the scores in college entrance examination.

Data Processing. The part of the data in database is shown in Table 1.

Table 1 Database part of the data sample

\begin{tabular}{|l|c|c|c|c|}
\hline Name & $\begin{array}{c}\text { Scores of } \\
\text { university } \\
\text { entrance } \\
\text { examination }\end{array}$ & $\begin{array}{c}\text { Average } \\
\text { achievement at } \\
\text { school }\end{array}$ & Scores & Average scores \\
\hline Wang* & 120 & 75 & 538 & 469 \\
\hline Zhao* & 117 & 77 & 546 & 460 \\
\hline Liang* & 126 & 80 & 520 & 531 \\
\hline Xiao* & 102 & 71 & 499 & 450 \\
\hline An* & 111 & 73 & 512 & 428 \\
\hline Ren* & 105 & 67 & 521 & 449 \\
\hline Hao* & 121 & 78 & 498 & 427 \\
\hline Liu* & 98 & 66 & 500 & 449 \\
\hline Xie* & 115 & 75 & 569 & 527 \\
\hline Feng* & 95 & 74 & 496 & 413 \\
\hline
\end{tabular}


The dispersing processing of data is performed below. According to the university performance evaluation standards and different scores, they are divided into excellent, fine, medium, poor, inferior and other grades, as shown in Table 2.

Table 2 Segmentation level of scores

\begin{tabular}{|c|c|c|c|c|}
\hline \multicolumn{5}{|c|}{ Segmentation Level of Scores } \\
\hline Excellent & Fine & Medium & Poor & Inferior \\
\hline $90-100$ & $80-89$ & $70-79$ & $60-69$ & $0-59$ \\
\hline
\end{tabular}

The database $D$ is scanned to get a new database $D^{\prime}$, and the converted data is shown in Table 3 .

Table 3 Converted database

\begin{tabular}{|c|c|c|c|c|}
\hline Identification $T$ & $X_{1}$ & $X_{2}$ & $X_{3}$ & $X_{4}$ \\
\hline$T_{1}$ & 1 & 1 & 1 & 1 \\
\hline$T_{2}$ & 1 & 1 & 1 & 1 \\
\hline$T_{3}$ & 1 & 0 & 1 & 1 \\
\hline$T_{4}$ & 1 & 1 & 1 & 1 \\
\hline$T_{5}$ & 0 & 0 & 0 & 0 \\
\hline
\end{tabular}

\section{Summary}

With the new media era is coming, the comprehensive analysis in all aspects of people's life has infiltrated a variety of new media tools and platforms, which greatly affected the students' learning and life. It is also beneficial to the innovation of electronic technology teaching in colleges and universities. The teachers should actively apply the convenient new media conditions, break through the constraints of traditional teaching mode, and carry out constant innovation. With the openness of the new media, the communication with learners in different regions of the world should be strengthen to look for a new way of electronic technology teaching in the process of continuous learning and reference. At the same time, we must pay attention to the research of outcomes-based education platform, integrate all kinds of teaching resources, promote resource sharing, enrich outcomes-based education methods, and strengthen the interest of electronic technology teaching.

\section{References}

[1] A. Ravenscroft, A. Schmidt, J. Cook, and C. Bradley. Designing Social Media for Informal Learning and Knowledge Maturing in the Digital Workplace, Journal of Computer Assisted Learning, 28 (2012), 112-120.

[2] JY Nancy, NH Khanna, and K Arputharaj. A Q-back Propagated Time Delay Neural Network for Diagnosing Severity of Gait Disturbances in Parkinson's Disease, Journal of Biomedical Informatics, 60 (2016), 169-176.

[3] BBC Onwuagboke, and TKR Singh. Effects of Computer Self-Efficacy on Pre-Service Art Teachers' Achievement in Graphic Design, Pertanika Journal of Social Science \& Humanities, 24 (2016), 1315-1333.

[4] BR Webb, and SB Gallagher. Mapping Commonalities and Differences in Software Engineering and Graphic Design Approaches to Multimedia Systems Development", Journal of Computer Information Systems, 46 (2016), 87-98.

[5] S Laing, and M Masoodian. A Study of the Influence of Visual Imagery on Graphic Design Ideation, Design Studies, 45 (2016), 187-209.

[6] S Narayanakumar, and K Raja. A BP Artificial Neural Network Model for Earthquake Magnitude Prediction in Himalayas, Circuits \& Systems, 7 (2016), 3456-3468. 
[7] C Bergmeir, and JM Benitez. Neural Networks in R Using the Stuttgart Neural Network Simulator: RSNNS, Carpathian Journal of Electronic \& Computer Engineering, 46 (2016), 3-6.

[8] A Graves, G Wayne, M Reynolds, T Harley, and I Danihelka. Hybrid Computing Using A Neural Network with Dynamic External Memory, Nature, 538 (2016), 471.

[9] M Hernández-Pajares, JM Juan, and J Sanz. Neural Network Modeling of the Ionospheric Electron Content at Global Scale Using GPS Data, Radio Science, 32 (2016), 1081-1089.

[10] A Shafiee, A Nag, N Muralimanohar, R Balasubramonian, and JP Strachan. ISAAC: A Convolutional Neural Network Accelerator with In-Situ Analog Arithmetic in Crossbars, Acm Sigarch Computer Architecture News, 44 (2016), 14-26. 